\title{
Improved EM-Based Tissue Segmentation and Partial Volume Effect Quantification in Multi-sequence Brain MRI
}

\author{
Guillaume Dugas-Phocion ${ }^{1}$, Miguel Angel González Ballester ${ }^{1}$, \\ Grégoire Malandain ${ }^{1}$, Christine Lebrun $^{2}$, and Nicholas Ayache ${ }^{1}$ \\ 1 Inria, Epidaure Project Team - 2004 route des Lucioles - BP 93 \\ 06902 Sophia Antipolis Cedex, France, \\ Guillaume.Dugas_Phocion@sophia.inria.fr, \\ http://www-sop.inria.fr/epidaure \\ 2 Pasteur Hospital, Neurology Department \\ 30 voie romaine BP 69, 06002 Nice, France
}

\begin{abstract}
The Expectation Maximization algorithm is a powerful probabilistic tool for brain tissue segmentation. The framework is based on the Gaussian mixture model in MRI, and employs a probabilistic brain atlas as a prior to produce a segmentation of white matter, grey matter and cerebro-spinal fluid (CSF). However, several artifacts can alter the segmentation process. For example, CSF is not a well defined class because of the large quantity of voxels affected by the partial volume effect which alters segmentation results and volume computation. In this study, we show that ignoring vessel segmentation when handling partial volume effect can also lead to false results, more specifically to an over-estimation of the CSF variance in the intensity space. We also propose a more versatile method to improve tissue classification, without a requirement of any outlier class, so that brain tissues, especially the cerebro-spinal fluid, follows the Gaussian noise model in MRI correctly.
\end{abstract}

\section{Introduction}

The segmentation of pathological tissues in multi-spectral MRI is useful, for example for diagnosis purpose. The intensity signature of healthy tissues is more predictable than the one of potential lesions: having a good characterization and segmentation of healthy tissues is the first step to separate them from lesions.

For this task, the Expectation Maximization (EM) framework [1 is a popular tool. It provides a segmentation of MRI into three classes: grey matter, white matter, cerebro-spinal fluid (CSF). However, multiple artifacts affect the segmentation results. As an example, voxels at the interface between two tissues contain more than one tissue: this is called Partial Volume Effect (PVE) [2]. Furthermore, potential lesions are not handled here. Some solutions have been proposed in the literature to overcome these problems.

Lesions can be separated from healthy tissues by adding an outlier class, without any prior in the intensity space [3]. In [4], a tissue classification is performed and PVE voxels are labeled in a post-processing step. Another way to 
take PVE into account is to segment PVE voxels in the EM framework as a class, the parameters of which are constrained from other pure classes [5]. These PVE models have only been tested on T1 MR images though. In this study, we show that the CSF class is not well defined in the multi-spectral T2/PD MRI intensity space. Even with a PVE model such as [5], the segmentation still suffers from an over-estimation of the variance of each class, especially the CSF. We propose a method to deal with this problem, which will lead to the segmentation of vessels, in addition of other brain tissues. We validate our study on a database of T2/PD MRI of 36 multiple sclerosis patients and 9 healthy subjects, showing a significant decrease of the variance of pure classes.

\section{EM-Based Segmentation of Brain Multi-spectral MR Images}

Before performing any segmentation, we apply a skull-stripping technique to the images to keep brain tissues only. Many methods are available in the literature, our method is closely related to the one described in [6]. We first generate a primary EM-based segmentation that we refine with mathematical morphology. More details can be found in [7].

\subsection{Basic Algorithm}

MRI noise is known to follow a Rician probability density function (PDF). Since a Rician PDF, if mean value is greater than 0 , can be reasonably approximated by a Gaussian PDF, it is acceptable to say that noise in multi-spectral MR images follows a Gaussian PDF. As a consequence, the segmentation is based on a Gaussian mixture model within the intensity space. Each class $k$ is represented by the regular Gaussian parameters in the intensity space: mean $\mu_{k}$ and covariance matrix $\Sigma_{k}$. The EM algorithm consists in iterating two steps: labeling of the image - Expectation step - and estimation of the class parameters by maximizing the likelihood of the whole image - Maximization step [1].

The labeling is the computation of the probability of a tissue class given the image and the parameters. The application of Bayes' rule gives the solution to the problem, summarized in equation 11, $l_{j}$ and $y_{j}$ are respectively the label and the multi-spectral intensity of the voxel $j$; Y is the whole image and $\Phi$ is the set of parameters. $\Pi_{l_{j}=a}$ is the a priori probability to get the label $a$ for the voxel $j$.

$$
p\left(l_{j}=a \mid Y, \Phi^{m-1}\right)=\frac{p\left(y_{j} \mid l_{j}=a, \Phi^{(m-1)}\right) \Pi_{l_{j}=a}}{\sum_{k} p\left(y_{j} \mid l_{j}=k, \Phi^{(m-1)}\right) \Pi_{l_{j}=k}}
$$

$\Pi_{l_{j}}$ is different whether a probabilistic atlas is available or not. When no atlas is used, the prior does not depend on the position $j$ of the voxel $\left(\Pi_{l_{j}=a}=\Pi_{a}\right)$ and needs to be re-estimated at each iteration (by $\Pi_{a}=\sum_{j} p\left(l_{j}=a \mid Y, \Phi^{m-1}\right) / \sum_{j}$ ) for convergence purposes. When the atlas is available, the spatially dependent prior simply needs to be normalized: $\forall j, \sum_{k} \Pi_{l_{j}=k}=1$. 
The estimators that maximize the likelihood are the MAP estimators corresponding to the mean and covariance matrix. As an approximation, we use the parameters of the last iteration $\Phi^{(m-1)}$ when computing the parameters at iteration $m$. Both estimators are represented within equations 2 and 3

$$
\begin{gathered}
\mu_{k}^{m}=\frac{\sum_{j} p\left(l_{j}=k \mid Y, \Phi^{(m-1)}\right) y_{j}}{\sum_{j} p\left(l_{j}=k \mid Y, \Phi^{(m-1)}\right)} \\
\sum_{k}^{m}=\frac{\sum_{j} p\left(l_{j}=k \mid Y, \Phi^{(m-1)}\right)\left(y_{j}-\mu_{k}^{m}\right)\left(y_{j}-\mu_{k}^{m}\right)^{\prime}}{\sum_{j} p\left(l_{j}=k \mid Y, \Phi^{(m-1)}\right)}
\end{gathered}
$$

\subsection{Results of the Basic Algorithm}

This method was applied on T2/PD $(\mathrm{TE}=8 / 104, \mathrm{TR}=5000, \mathrm{ETL}=16$, voxel size: $\left.0.937^{*} 0.937^{*} 2\right) \mathrm{MR}$ images, from an acquisition protocol concerning 36 relapsing remitting multiple sclerosis patients. Three classes were segmented: white matter, grey matter and CSF, with the corresponding atlas for each class, obtained by affinely registering the MNI probabilistic atlases [8]. It takes less than 30 seconds for $256^{*} 256^{*} 64$ images on a $3 \mathrm{Ghz}$ PC. The results are shown in figure 1

Visual inspection of white matter segmentation seems satisfactory. The grey matter Mahalanobis ellipse is deformed towards the CSF ellipse, mostly because of the partial volume effect, and the CSF class has a tremendously high variance, which seems quite hard to explain with these PVE voxels only, as shown next.

\section{Improved Tissue Segmentation}

\subsection{Partial Volume Effect (PVE)}

MR images have a limited resolution. Voxels at the interface between two classes, e.g. grey matter and CSF, have an intermediate intensity depending on the proportion of tissues in this voxel. Those "PVE voxels" will bias a segmentation into pure class voxel since it invalidates the Gaussian mixture model for the whole image.

Let there be two tissues $a$ and $b$, their intensities $y_{a}$ and $y_{b}$ respectively, and $\alpha$ the proportion of tissue $a$ in the considered PVE voxel. Let $N(\mu, \Sigma)$ be the Gaussian PDF with parameters $\mu$ and $\Sigma$. Both $y_{a}$ and $y_{b}$ follow a Gaussian PDF, respectively $N\left(\mu_{a}, \Sigma_{a}\right)$ and $N\left(\mu_{b}, \Sigma_{b}\right)$. The intensity of this voxel $y_{\mathrm{PVE}}$ is a random variable that can be computed using $y_{\mathrm{PVE}}=\alpha y_{a}+(1-\alpha) y_{b}$.

If we consider PVE voxels only, $\alpha$ is uniformly distributed. Separating PVE voxels from pure voxels is not trivial [2]. However, if $\alpha$ is fixed and known, $y_{\mathrm{PVE}}$ follows a Gaussian PDF with the following parameters:

$$
y_{\mathrm{PVE}} \sim N\left(\alpha \mu_{a}+(1-\alpha) \mu_{b}, \alpha^{2} \Sigma_{a}+(1-\alpha)^{2} \Sigma_{b}\right)
$$

One solution is to emulate the partial volume effect by a fixing $\alpha$ to some constant values, and computing the PVE classes within the regular EM. The 

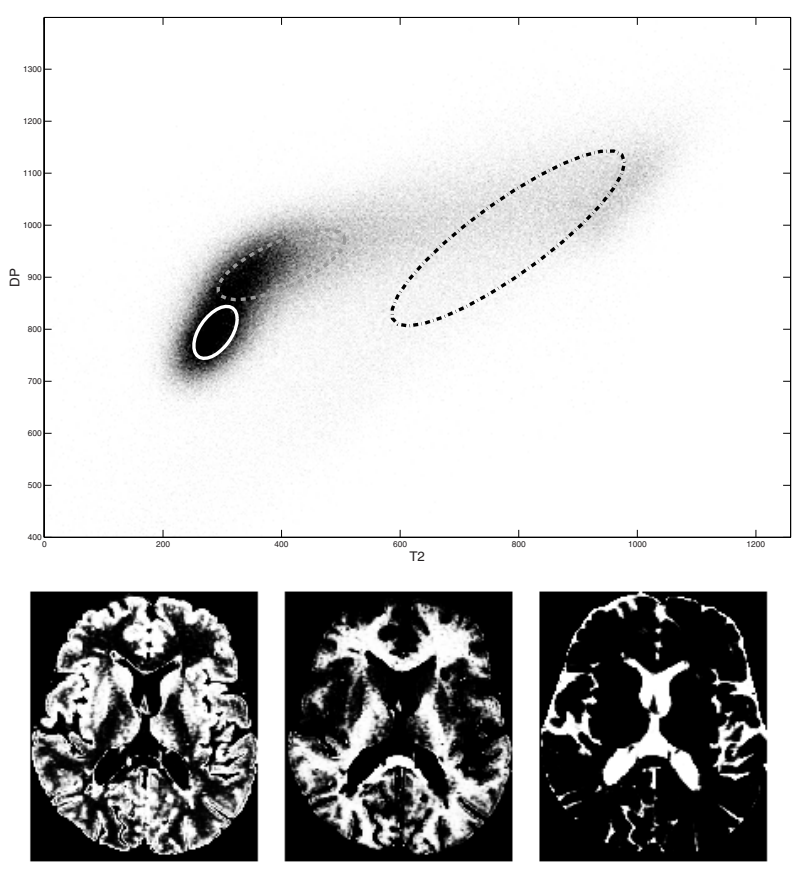

Fig. 1. Joint histogram between T2 and PD MRI, representation of tissue classes using the Mahalanobis ellipse and the corresponding classification. Three classes are segmented, corresponding to the three atlas classes (from left to right): grey matter (grey dashed), white matter (white), and CSF (black dash-dotted).

algorithm will consist in iterating three steps: labeling of all classes (including PVE classes), estimation of class parameters only for pure classes - white matter, grey matter and CSF -, and computing PVE classes parameters using equation 4 This method is interesting as it remains easy to compute and respects the original EM framework [9]. Some results are shown in figure 2 .

As we can see, the grey matter class has a reasonable variance now, comparable to the one of the white matter. Indeed, adding PVE classes (between the grey matter and the CSF) allowed to decrease the variance along the T2 axis where both classes are well separate. However, the variance of the CSF remains at very high values, and the direction of the main axis shows that this problem cannot be solved solely by addressing the partial volume effect between the former segmented tissues.

\subsection{Introducing a Fourth Class}

As demonstrated above, the PVE does not explain the huge variance of the CSF class. A close look at the images (see figure 4, c, 4,i) shows that some dark structures (identified mainly as vessels) are also classified as CSF. Those structures 


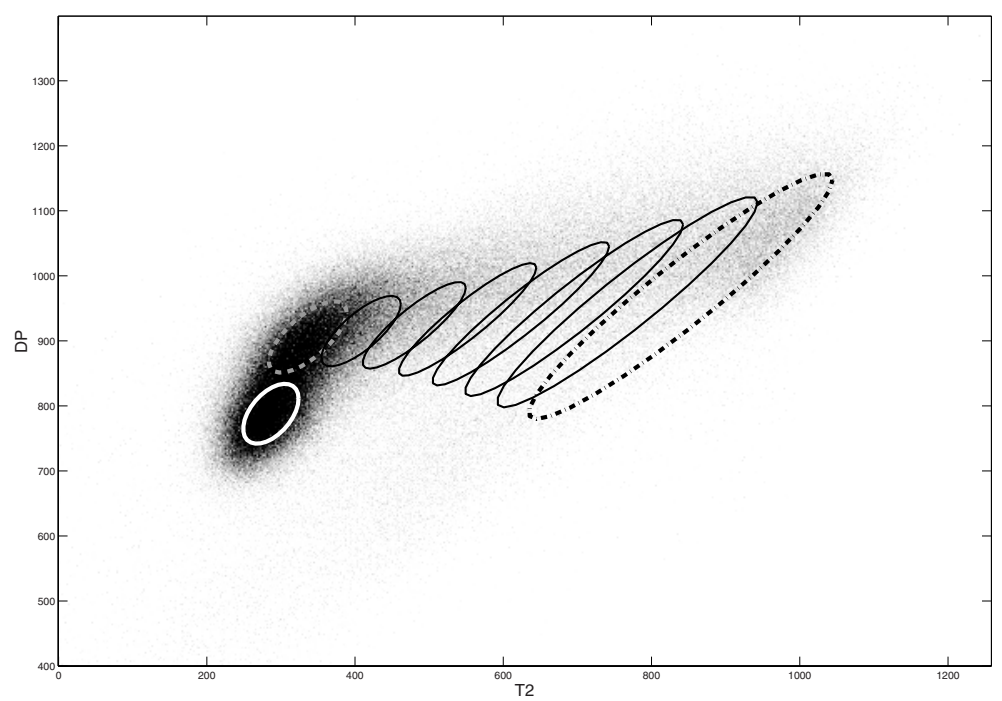

Fig. 2. Joint histogram between T2 and PD MRI, with PVE between grey-matter and CSF. 4 PVE classes were used $(\alpha \in\{0.2,0.4,0.6,0.8\})$. The variance of the grey matter class has been reduced, but the CSF class is still oversized.

are not guaranteed to be labeled as outliers, if an outlier class is added as in [3]. Thus, we prefer to introduce explicitely a vessel class.

In the three class EM, vessels are classified as CSF. This CSF class has to be splitted into two classes, vessels and CSF. To achieve this, we will define

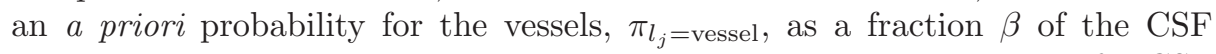
probability given by the atlas, $\Pi_{l_{j}=\mathrm{CSF}}$. With this new class, the prior for CSF becomes $\pi_{l_{j}=\mathrm{CSF}}=(1-\beta) \Pi_{l_{j}=\mathrm{CSF}}$.

The labeling can then be achieved into an EM framework. Since the relative ratio of vessels with respect to CSF is not known, $\beta$ has to be estimated at each iteration for convergence purposes by

$$
\beta=\frac{\sum_{j} p\left(l_{j}=\operatorname{vessel} \mid Y, \Phi\right)}{\sum_{j} p\left(l_{j}=\operatorname{vessel} \mid Y, \Phi\right)+\sum_{j} p\left(l_{j}=\mathrm{CSF} \mid Y, \Phi\right)}
$$

By introducing this fourth class, the system becomes more flexible, and still allows the adaptation of the algorithm to unforeseen tissues (e.g. with an outlier class) without loosing the information from the atlas.

The final algorithm becomes the following:

1. compute a labeling for each class (Expectation Step);

2. estimate parameters for the following classes: white matter, grey matter, CSF and vessels;

3. compute analytically PVE classes parameters using equations 4. 
4. recognize the CSF class by selecting the class with highest mean on T2 axis (the CSF class has to be distinguished from the vessel class for the PVE computation with the grey matter).

Results are presented in figures 3 and 4. The CSF class only includes pure CSF (compare figure 4.i and 4.j). Its variance has decreased by a large amount, and is now comparable to grey matter and white matter class variances, which corroborates a Gaussian noise model for the MR signal.

Introducing the vessel class allows also a better recognition of PVE voxels between CSF and grey matter: Mahalanobis ellipses of PVE voxels distributions in figure 3 are now well separated.

It is important to point out that the simple usage of an outlier class may fail to separate the CSF from vessels here. Indeed, both classes have a similar number of voxels, and outlier detection assumes that the outlier class is less represented than the main class.

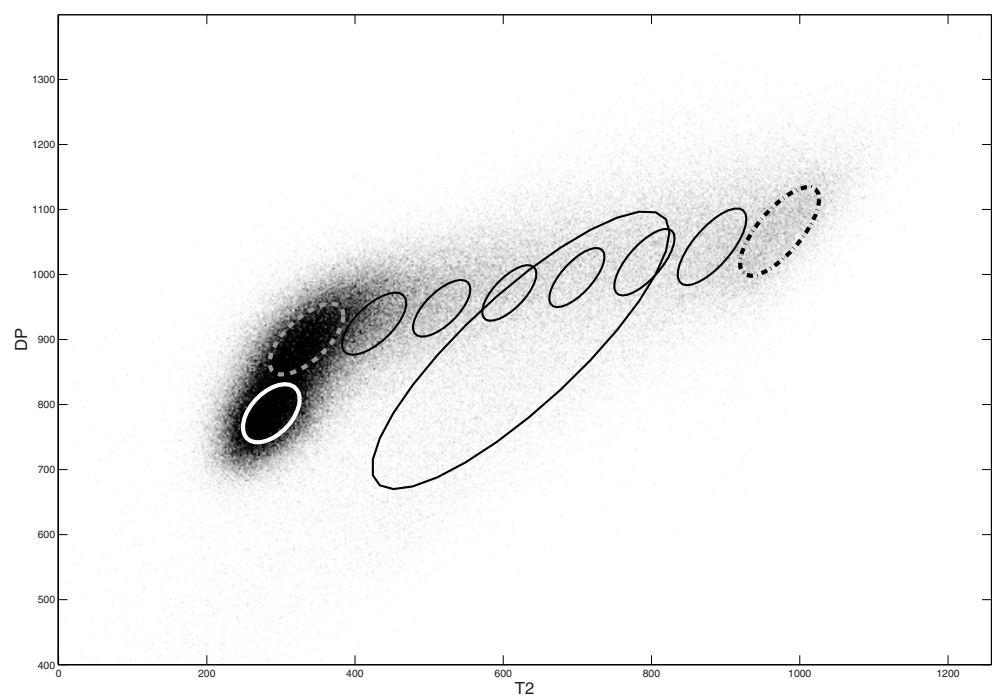

Fig. 3. Joint histogram between T2 and PD MRI, and representation of tissue classes using the Mahalanobis ellipse. As one class, which prior is a fraction of CSF atlas, is added, the CSF class does not include vessels anymore: the main three brain tissues grey matter, white matter and CSF - follow the Gaussian noise model properly.

\section{Conclusion and Future Work}

Our main finding is that introducing explicitely the vessel class into the EM framework allows a significant improvement in the classification.

The system has been tested on 36 multiple sclerosis patients MRI and 9 healthy subjects, with a good estimation of the parameters, reflected by a clear 

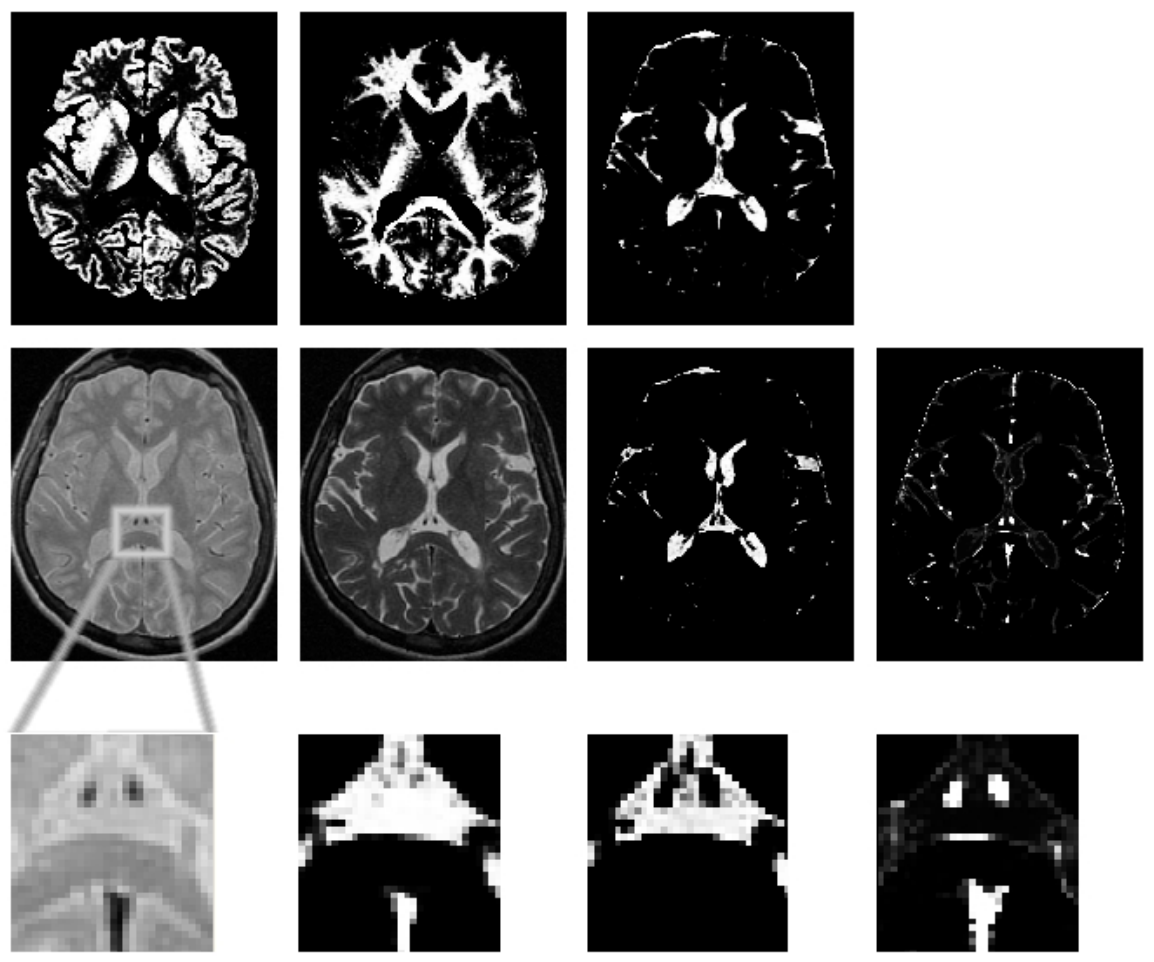

Fig. 4. Final segmentation: grey matter (a), white matter (b), CSF without vessel class (c), T2 MRI(d), Proton Density MRI (e), CSF (f), other class including vessels (g). Zoom : Proton Density (h), CSF without vessel class (i), CSF (j), other class including vessels $(\mathrm{k})$.

Table 1. Estimation of T2 standard deviation for brain tissues, resulting on experiments over 36 patients and 9 healthy subjects. Notice that CSF standard deviation has been divided by 3 with the vessel class, without any intensity non-uniformity correction.

\begin{tabular}{l|ccc} 
T2 std deviation & Grey matter & White matter & CSF \\
\hline no PVE & 68.5 & 32.0 & 160.2 \\
PVE without vessels & 49.7 & 33.9 & 167.9 \\
PVE + vessels & 46.7 & 34.1 & 58.3
\end{tabular}

\begin{tabular}{l|ccc} 
PD std deviation & Grey matter & White matter & CSF \\
\hline no PVE & 53.3 & 41.8 & 145.2 \\
PVE without vessels & 49.9 & 41.2 & 159.6 \\
PVE + vessels & 49.7 & 40.4 & 60.5
\end{tabular}


decrease of variance for CSF class, as shown in table 1 The T2 CSF standard deviation has been divided by 3 , and never exceeds 65 in all the images, although it exceeds 150 with a simple PVE model. A closer look at the final segmentation indicates that the new segmentation of CSF does not include neither vessels nor partial volume effect, that are this time correctly extracted. The whole process is still quite fast, since we simply added a few classes in the initial EM framework: it takes around 4 minutes to run the system on a $3 \mathrm{Ghz}$ PC.

One application of this method may be the computation of volumes (CSF volume, or brain atrophy) since it allows a more accurate estimation of probabilities for each tissue within each voxel. Brain atrophy is indeed a good disease indicator, in Alzheimer disease or multiple sclerosis for example.

The segmentation of pathological tissues (multiple sclerosis lesions or tumors), our primary motivation, is also an interesting derived application. We argue that this may be facilitated with a good classification of healthy tissues, as the one proposed in this study. This is left as future work.

\section{References}

1. Dempster, A., Laird, N., Rubin, D.: Maximum likelihood from incomplete data via the EM algorithm. Journal of the Royal Statistical Society 39 (1977) 1-38

2. González Ballester, M.A., Zisserman, A., Brady, M.: Estimation of the partial volume effect in MRI. Medical Image Analysis 6 (2002) 389-405

3. Leemput, K.V., Maes, F., Vandermeulen, D., Colchester, A., Suetens, P.: Automated segmentation of multiple sclerosis lesions by model outlier detection. IEEE TMI 20 (2001) 677-688

4. Shattuck, D.W., Sandor-Leahy, S.R., Shaper, K.A., Rottenberg, D.A., Leahy, R.M.: Magnetic Resonance Image Tissue Classification Using a Partial Volume Model. NeuroImage 13 (2001) 856-876

5. Noe, A., Gee, J.: Efficient Partial Volume Tissue Classification in MRI Scans. In: Proc. of MICCAI. Volume 2488 of LNCS., Springer (2002) 698-705

6. Kapur, T., Grimson, W.E., Wells, W.M., Kikinis, R.: Segmentation of brain tissue from magnetic resonance images. Med Image Anal 1 (1996) 109-127

7. Dugas-Phocion, G., González Ballester, M.A., Lebrun, C., Chanalet, S., Bensa, C., Malandain, G., Ayache, N.: Hierarchical Segmentation of Multiple Sclerosis Lesions in Multi-Sequence MRI. In: International Symposium on Biomedical Imaging. (2004)

8. Collins, D., Zijdenbos, A., Kollokian, V., Sled, J., Kabani, N., Holmes, C., Evans, A.: Design and construction of a realistic digital brain phantom. IEEE TMI 17 (1998) 463-468

9. Ruan, S., Jaggi, C., Xue, J., Fadili, J., Bloyet, D.: Brain tissue classification of magnetic resonance images using partial volume modeling. IEEE Trans Med Imaging 19 (2000) 1179-1187 